\title{
Empatía y tacto pedagógico profesional: ¿un saber pedagógico y humanista?
} Empathy and Professional Pedagogical Tact: A Pedagogical Knowledge?

Susanne Müller-Using ${ }^{l}$ Universidad de Osnabrüick

Alemania

\section{Resumen}

Esta contribución examina la importancia educacional de la empatía, con base en el estado del arte de la investigación interdisciplinaria. La causa de esta discusión es la percepción de una falta de empatía vivida en la sociedad. La metodología aplicada se fundamenta en soportes científicos e investigaciones del comportamiento de las personas. Más aún, este trabajo trata de la experiencia de ser un educador y, por consiguiente, de la tarea que implica trabajar con niños y adolescentes. Además, el artículo se enfoca en la discusión del cómo y el porqué de la empatía en la personas, tomando en cuenta la historia y la actualidad. Además habla de cómo la empatía podría ser un elemento importante en cuestiones pedagógicas de la educación en valores para niños y adolescentes, ya que la empatía incrementa la confianza en el propio proceso de aprendizaje que fortalece la confianza en el marco de las relaciones interpersonales. Asimismo, el artículo describe el tacto pedagógico y la empatía como actitudes que se complementan y resultan igual de importantes para el desarrollo, aprendizaje y la formación de un niño.

Palabras clave: tacto pedagógico; humanismo; educación; empatía.

$1 \quad$ Experta en la teoría educacional sobre cultura-comparativa, Especialista en estudios culturales y religiosos en una perspectiva comparativa. Directora Científica del núcleo de investigación del Centro Costa Rica de la Universidad de Osnabrück. Correo electrónico: susanne.mueller-using@uni-osnabrueck.de 


\begin{abstract}
This contribution examines the educational importance of empathy, based on the current situation of interdisciplinary research. The cause of this discussion is the perception of a lack of empathy experienced in society. The methodology applied is based on scientific support and research into people's behavior. Moreover, this work addresses the experience of being an educator and, consequently, the task of working with children and adolescents. In addition, the article focuses on the discussion of how and why people experience empathy, taking into account history and current events. It also talks about how empathy could be an important element in pedagogical issues of education in values for children and adolescents since empathy increases confidence in the own learning process that strengthens confidence within the framework of interpersonal relations. The article also describes the pedagogical tact and empathy as attitudes that complement each other and are equally important for a child's development, learning, and upbringing.
\end{abstract}

Keywords: pedagogical touch; humanism, education empathy

\title{
Estado actual de investigación: ¿qué significa empatía y qué sabemos de ella?
}

De momento, se discute cada vez más sobre el tema de la empatía. La causa de esa discusión es la percepción de una falta de empatía vivida en la comunidad y la sociedad. Por ejemplo, en el contexto de la crisis financiera, según De Waal (2009) como reacción ante el comportamiento sin miramientos o escrúpulos de banqueros y especuladores de bolsa, atribuido a los actos impasibles de los actores quienes enfocaron la maximización del beneficio a corto plazo como única meta, en contra de todo sentido común y a costa de los demás. También las condiciones previas que favorecen tal comportamiento se estaban y se están criticando y discutiendo en público. La búsqueda en la base de datos de literatura FIS Bildung ha demostrado que las entregas sobre la empatía se triplicaron entre el 2000 y el 2010. En el 2018, todavía son cinco veces más entregas que en el 2000. Los motivos de la tematización residen, entre otras cosas, en una más alta disposición a la violencia, sobre todo en el ámbito de la ciberviolencia, el tal cybermobbing (Pfetsch, Müller y Ittel, 2014). Otros motivos son casos como el de los ataques en el metro de Múnich o bien los ataques amok en Winnenden 2009 y Utøya 2011, así como el susto y el choque ante la frialdad masiva por parte de los delincuentes, lo que provocó una ola enorme tanto de consternación como de conmoción y también la pregunta sobre el porqué.

Alemania se encuentra en segundo lugar, directamente detrás de los EE.UU., en cuanto a las víctimas y personas heridas en los ataques amok (Nano Spezial, 2014). 
Para el discurso científico, es esencial hacer posible un análisis y una definición más detallados de lo que caracteriza la empatía con base en la investigación actual. Así, los conceptos de Einfühlung y Einfühlungsvermögen, los significados originales de empatía, pueden ser descritos de manera más diferenciada y en su estado de proceso entre emoción y cognición. Esto se hace cada vez más de acuerdo con la investigación y el diálogo interdisciplinarios (Singer y Bolz, 2013).

Actualmente, la discusión científica interdisciplinaria sobre la empatía se mantiene en las áreas de las ciencias del comportamiento (De Waal, 2009 en Rizzolatti y Sinigaglia, 2008), la psicología del desarrollo (Bischof-Köhler, 2011), la medicina (Aust, 2013), las neurociencias (Singer y Bolz, 2013; Keysers, 2012; Bauer, 2005), así como en las ciencias humanas y sociales (Breyer, 2013; Rifkin, 2012; Breithaupt, 2009).

Las preguntas enfocan, por ejemplo, el significado de la empatía referente a la historia de la civilización y, con esto, su acepción social. El sociólogo Rifkin (2012) expone la historia de la civilización humana como una de la evolución de la empatía, en la cual concluye que el Homo sapiens siempre ha sido un Homo empathicus. Siempre que, en la historia de la humanidad, se aplicaban los valores de empatía, cohesión y apoyo para los demás, el resultado era generalmente un cambio positivo hacia el desarrollo. Por eso, Rifkin describe la empatía como uno de los rasgos humanos más fundamentales. El investigador conductista Frans De Waal (2009) subraya esta observación al diseñar la empatía como hilo conductor en la historia de la evolución humana.

Más concretamente, se trata de las posibilidades humanas y los límites de la Einfühlung o de la capacidad de identificar los sentimientos y ponerse en el lugar del otro (Mayer, 2013, p. 109). En el contexto de las relaciones sociales, la cuestión de los límites y de la falta de empatía está estrechamente ligada a la de cómo se desarrolla e impulsa.

En el contexto alemán se analizó que aproximadamente $14 \%$ de los alemanes (más de uno de cada diez) es alexitímico, es decir, incapaz de captar las emociones de los otros. Eso es lo que descubrió Isabella Heuser, directora de la clínica de psiquiatría y psicoterapia de la Berliner Charité y profesora que dirige el polo de excelencia Languages of Emotion en la Freie Universität Berlin, promovido por la Fundación Alemana para la Investigación Científica (DFG), en un amplio estudio (según la revista Wissenschaftsmagazin Fundiert del 12 de junio del 2008). Alexitimia es la incapacidad de leer los sentimientos de los demás y de expresar los propios. De acuerdo con Heuser, esa condición no se considera enfermedad, 
sino un rasgo característico de las personas afectadas. Lo que es característico de los alexitímicos es su objetividad acentuada y su sobriedad en palabras a la hora de hablar de sentimientos y, muchas veces, hay indicios de descuido emocional. Dado que la percepción de sentimientos, los propios y los de otros, es un elemento importante de la empatía, estos resultados de investigación no son insignificantes para la discusión que sigue.

¿Pero cómo definimos exactamente la empatía en la actualidad? Como lo indica el diccionario etimológico, el término se remonta a Rudolf Hermann Lotze (1817-1881), quien utiliza el préstamo del griego para traducir la palabra alemana Einfühlung. Más tarde, el término es probablemente introducido en el vocabulario inglés por el psicólogo inglés Edward Bradford Titchener (1867-1927) cuando tradujo el término Einfühlungsvermögen de las obras de Theodor Lipps.

Según el diccionario Brockhaus Wissensservice (2014), empatía se refiere a la disposición y capacidad de ponerse en el modo de vivir de otra persona y de percibir su estado emocional y de ánimo. Hay que añadir que tal comportamiento requiere la capacidad de experimentar las propias emociones, así como reconocer y calificar los estados afectivos de los otros.Doris Bischof-Köhler (2011), en su definición de la empatía, indica que por el lado fenomenal corresponde a la experiencia de experimentar de manera directa el estado emocional o bien la intención del otro y así comprenderlos. Agrega que pese a esa participación, el sentimiento o bien la intención queda claramente como propio de la otra persona.Por lo tanto, elementos centrales de la empatía son la percepción del comportamiento expresivo del otro, al igual que percibir su situación y la capacidad, relacionarla con esta, de tomar la perspectiva y el rol de ese otro. La empatía tiene un componente tanto emocional como cognitivo. Según Bischof-Köhler, sin embargo, es principalmente la calidad emocional propia de la compasión empática que hace posible la comprensión.

En la opinión de Tania Singer, empatía es el mero momento de participar en el estado emocional del otro (Singer y Bolz, 2013). Para ella, la cuestión más fascinante es saber qué influye en que ese momento sea convertido en compasión o en alegría del mal ajeno y de qué depende la decisión a favor o en contra de una conducta prosocial. Podrían ser relevantes, también, aspectos pedagógicos de la educación en valores para niños y adolescentes. Esta última definición de la empatía muestra que la característica tiene, sin duda, resonancia, pero, por sí sola, no está dirigida de manera automática al bienestar del otro. Por otro lado, la empatía es una condición previa necesaria para la conducta prosocial y altruista (Batson, Duncan, Ackerman, Buckley y Birch, 1981). Empathy-Altruism-Hypothesis: el 
cuidado empático nace tomando la perspectiva del otro de manera imaginaria y puede incitar a acciones altruistas y prosociales.

\section{1. ¿Cuál es el significado pedagógico de la empatía?}

Lo genuinamente pedagógico comprende todo lo que caracteriza las pedagogías. Su clasificación, análisis, investigación y renovación son, según Paschen (2005), el objeto de la ciencia de la educación. Si se pudiera mostrar que la empatía es un elemento fijo de lo que se considera genuinamente pedagógico, sería un objeto potencial de la ciencia de la educación y así también de importancia pedagógica. Refiriéndose a Heinrich Roth (1906-1983), Wolfgang Nieke introduce la competencia de acción como término genuinamente pedagógico en el discurso educativo (Nieke, 2002) y relaciona competencias pedagógicas centrales, como conocimientos profesionales y especializados, competencia metodológica, competencia personal y competencia social, con las tareas de análisis de la sociedad, diagnóstico situacional y autorreflexión.

Prange (2005) introduce la estructura de mostrar de la educación como algo genuinamente pedagógico o, en otras palabras, el arte de la introducción a campos del saber que comprenden la capacidad de despertar el interés de niños y adolescentes por temas seleccionados, así como de acercarles de manera didácticamente reflexionada al razonamiento con los campos del saber relevantes para la vida comunitaria (triángulo pedagógico). Referente al contexto escolar (no solo en él, pero en él en particular) es igual de importante incrementar la confianza en el propio proceso de aprendizaje que fortalecer la confianza en el marco de las relaciones interpersonales, entre los profesores y los alumnos, al igual que en los alumnos entre sí (acerca de la importancia de la confianza en la vida escolar (Schweer y Padberg 2002). Confiar (¡en sí mismo!) es un fundamento esencial para el aprendizaje y se considera una necesidad básica del hombre. Confianza requiere seguridad y esta el respeto de la dignidad del niño y el trato respetuoso en las relaciones interpersonales (entre adultos, adultos y niños y niños entre sí). Un aspecto clave del compromiso formativo público, pero también de la mayoría de las pedagogías, es introducir a los niños y adolescentes a los fundamentos y las conductas del trato respetuoso entre los hombres, tanto como realizarlo de manera pedagógica para las diferentes edades (KMK, 2014; Hentig, 2009).

Una condición previa para cumplir todos estos requisitos y tareas es que los educadores y profesores hayan desarrollado empatía y que estén formados tanto para promoverla como para ayudar a niños y adolescentes a adquirirla. 
Así, los profesores se ven frente a un reto profesional para el que tienen que estar formados en un contexto académico. Además de los conocimientos de la materia, es necesario que aporten competencias de liderazgo creativas e inteligentes, pero también comunicativas y social-empáticas, para las que, por lo general, no están lo suficiente entrenados (Popp, 2012; Bertelsmann, 2007). Por eso hay que clarificar, en un marco teórico-profesional, cuáles son las competencias pedagógicas que deberían aportar los profesionales que trabajan en las instituciones, es decir, los profesores y también la dirección, para cumplir con estas tareas; asimismo, es preciso esclarecer cuál es la función de la empatía.

Parece crucial el argumento de que los niños suelen percibir y aprender de manera holística, que son socializados por su entorno y que se desarrollan lo mejor posible en determinadas condiciones con las que se enfrentan. De esto resulta que los pedagogos en las escuelas son responsables de dirigir los procesos pedagógicos de manera adecuada para niños, es decir de manera adaptiva y equilibrada, y así responder a los perfiles y necesidades individuales de los niños. Para poder actuar así, y esta opinión parece imponerse cada vez más, hay que ser empático (Kuhl Müller-Using y Solzbacher, 2011; Müller-Using, 2010, p. 35f; Bauer, 2006).

\section{Tacto pedagógico y empatía, ¿dos caras de la misma moneda?}

Hasta ahora, el discurso educativo ha prestado más atención al término del tacto pedagógico que a la empatía, dos términos afines. Introducido ya en el discurso pedagógico, Herbart (1776-1841) y discutido por Muth (1962) y Van Manen (1991, 1995ab) hasta hoy por su significado para el actuar pedagógico, el término tacto pedagógico se considera incluido en el discurso académico y es mucho más tematizado que la empatía. Pero, ¿en qué consisten las semejanzas y las diferencias en comparación con la empatía?

El desarrollo de la empatía, así como del tacto asegura la percepción del punto de vista del otro y la capacidad de dialogar. Ambos son esenciales para el actuar pedagógico y para animar la educación. Sin duda, el trato atento y respetuoso entre las personas es una condición previa central para la educación y el aprendizaje compartido. Según describe Jakob Muth, quien se apoya en Herbart y Schleiermacher, el tacto pedagógico es expresión de discreción y sensibilidad hacia los demás, especialmente hacia los alumnos / los que son educados. La sensibilidad la describe, refiriéndose a Martin Buber, como la conciencia para el "tú", para el otro, para su singularidad y sus derechos, y como respeto a la última inaccesibilidad del otro (Muth, 1962, p. 20). Igualmente, el estudioso explica el tacto como una forma de compasión que, bajo el término del tacto pedagógico, transfiere al 
proceso de la educación y que ve ligada al papel de ejemplo del profesor o educador en el actuar pedagógico.

Herbart (1887) consideró el tacto, o sea el tacto pedagógico, como elemento mediando entre teoría y práctica, ese que tiene en cuenta las partes que "la teoría pasa por alto" (Muth, 1962, p. 69) añade: "El tacto entra en los sitios que la teoría tuvo que dejar vacíos porque no son previsibles" (p.70). Según Muth y también Van Manen (1995), el tacto lleva consigo la capacidad de tomar decisiones y evaluar situaciones instantáneamente, lo que permite, por ejemplo, adaptar y ajustar las materias preparadas a las condiciones reales y la situación respectiva en clase.

Aunque Herbart y Muth pretendieron diferenciar tacto pedagógico y mera empatía, las semejanzas y los paralelismos son obvios.La discreción y el respeto que definen el tacto pedagógico se basan en la sensibilidad por el otro. Así lo describen sobre todo Muth y más tarde también Van Manen (1995). Desde Herbart hasta Van Manen se señala el tacto pedagógico como sentimiento y forma humanitaria en el trato interpersonal. En mi opinión, no obstante, el interés por el otro, por el de enfrente, así como la ocupación con el "tú" no se puede despertar sin la sensación empática, la capacidad de percibir el ritmo, como se diría en el lenguaje de la música y del tacto. Quizás es precisamente la sensación básica de la empatía que despierta la atención de la que crece el tacto. Desde esta perspectiva, el tacto pedagógico y la sensación empática no se distinguen fundamentalmente, sino son más bien la aplicación y la base como dos caras de la misma moneda, pues son dos aspectos del humanismo.

En cuanto a la implementación del tacto pedagógico, la sensación empática ofrece el motivo y permite tomar decisiones para la realización activa del actuar pedagógico en tiempo real. El ajuste del actuar pedagógico a las necesidades de los alumnos individuales, pero también de grupos enteros de alumnos, se hace mediante el procesamiento consciente de la sensación empática. Solo de ella se puede deducir después el actuar pedagógico. Ambos aspectos se basan en el momento de humanismo, comunidad y cuidado de los demás

Cuando el sentido "no planificable" del tacto está formado, entran en la rutina diaria de los educadores la discreción, el respeto y la sensibilidad (empatía) en el trato con niños y adolescentes (también con los padres y colegas), valores que definen el tacto y constituyen su base. Por lo que sabemos de ella, la empatía, como fundamento del humanismo y del sentido común, es la sensación profunda y la sensibilidad que se debe fomentar para entrenar y fortalecer la buena convivencia en sociedad, que forma una base sólida para aprender con éxito. Es bien conocido 
que la sensación en el sistema cognitivo es provocada por la percepción sensual (Fröhlich, 2002;Shi, 2011).

Por consiguiente, hay que tener en cuenta la percepción e integración multisensorial (impresión sensorial), en lo que se refiere al entrenamiento y fortalecimiento de la sensación empática. Este aspecto podría tener gran importancia en relación con la empatía y, en consecuencia, con el tacto en el trato con niños y adolescentes, así como con su transmisión pedagógica. Sin embargo, en la investigación educativa y el desarrollo de teorías, esta conexión apenas ha sido tematizada o investigada. En este sentido, juega un papel importante la percepción impecable mediante los cinco sentidos (la vista, el oído, el tacto, el gusto, el olfato) y otros que corresponden directamente con la experimentación de sentimientos, referentes al propio cuerpo, al entorno social y al grupo de referencia. Estos constituyen la base de los procesos cognitivos intelectuales (estética). Es cierto que este aspecto del conocimiento (Langer, 1987) ya se encuentra establecido en el discurso educativo a través de la estética. Pero en la formación docente recibe muy poca atención.

Si es cierto que, según dice Heuser (2008) en el estudio de la Charité de Berlín, más del $14 \%$ de los alemanes son alexitímicos, pues son incapaces de captar las emociones de los demás, en la mayor parte de los casos a causa de negligencia emocional (infantil) y en consecuencia del retroceso de los sentidos, resulta de esa situación, en mi opinión, una tarea pedagógica y de las ciencias educativas. Es esencial que, en los contextos pedagógicos profesionales y las instituciones de educación pública (formal y no formal), se tenga en cuenta y se aspire a que en instituciones públicas o reconocidas por el Estado, ya sean guarderías infantiles, escuelas, centros juveniles y centros de asistencia juvenil o universidades, los estudiantes sean atendidos por adultos formados para actuar de manera empática y con tacto pedagógico. Hay que asegurar que los niños sean tratados de manera respetuosa, sobre la base de una sensación empática saludable y con tacto pedagógico; que sean, entonces, "guiados" de manera pedagógica y que en este entorno puedan desarrollar una autoconciencia y una conciencia social saludables. También a los niños, cuyo desarrollo sensorial y cuya percepción ya están perjudicados, se les puede facilitar oportunidades educativas y de aprendizaje social e incluso se pueden recuperar ciertas etapas de su desenvolvimiento.Eso se hace mediante el actuar pedagógico que incluye los sentidos como físicos, sociales y cognitivos, al igual que les ayuda a desarrollar en habilidades sociales. Hoy en día, la necesidad es lamentablemente cada vez mayor (Myschker y Stein, 2014). 


\section{3. ¿Es posible asegurar o bien desarrollar la capacidad empática?}

La empatía y el tacto pedagógico son, entonces, aspectos esenciales del actuar pedagógico y han sido tratados repetidas veces en el discurso educativo/pedagógico. En especial, conciernen los modales en el trato profesional con niños y adolescentes. Como la empatía y el tacto pedagógico están estrechamente relacionados con el pensamiento, el sentimiento y el actuar pedagógico, probablemente se da por sentado; a saber, se les considera como base automatizada del actuar pedagógico, así que en el discurso profesional y público (salvo las excepciones ya tratadas como Muth y Van Manen), apenas se habla y se debate sobre ellos conscientemente. La suposición de que existen, o bien el hecho de que se les da por sentados por la cotidianidad laboral pedagógico, conduce fácilmente a que a estos aspectos no se les conceda mucha importancia en el nivel teórico-profesional. Aunque el valor de la empatía y del tacto pedagógico es indiscutible en cuanto a los conocimientos pedagógicos generales, está muy poco representado en el saber y discurso pedagógico, e. g. sobre la profesionalización de docentes.

La capacidad empática se muestra de manera bastante obvia en el contacto diario con los niños y se puede reconocer mediante observación directa. Por consiguiente, es evaluable, al menos por los que reconocen su valor para el actuar pedagógico y la estrategia pedagógica para dirigir. Voy a ilustrarlo con el siguiente ejemplo:

Hace unas semanas tuve la oportunidad de observar una situación en la que un grupo de estudiantes recibió a uno de diez niños en edad preescolar. El tema del día era "sonidos". Para saludarles y "calentarse", tenían planeado el juego "Preparo mi maleta" 2 , con la diferencia de que los niños debían enumerar e imitar "sonidos". Prepararon el espacio para sentarse en el piso solo después de que yo les hubiera preguntado. Sentados se explicó el juego. Al escuchar el nombre "Preparo mi maleta", los niños se emocionaron, porque lo conocían; pero, cuando se enteraron de la variante con los sonidos, no comprendieron por qué se deberían introducir "sonidos" en una maleta (ilógico) y se mostraron decepcionados. Poco después, siguieron las primeras intervenciones: "Eso es estúpido", "lo de los sonidos no es divertido" y "no tengo ganas de hacerlo". Las estudiantes se miraron muy desconcertadas, buscando ayuda, y no sabían qué decir. Los niños

2 Este juego requiere tener buena memoria. Todos los jugadores están sentados en corro. Empieza un jugador diciendo: "Preparo mi maleta y pongo zapatos". El siguiente tiene que memorizar lo que se dijo y añadir otra palabra. Con cada jugador, entra en el juego una palabra más. Hay que tener en cuenta que las palabras se enumeran en el orden correcto. Así, con cada jugador y con cada partida, se hace más difícil memorizarlo todo. Si un jugador dice una palabra incorrecta, la olvida o confunde el orden, pierde. Al final, queda el jugador con la mejor memoria y gana el juego. 
empezaron a retirarse y ya no participaron. Una de las estudiantes se dio cuenta, o bien sintió el alcance de la situación de que los niños estaban retirándose antes de que el juego hubiese empezado de verdad. Se acercó a los niños y dijo: "Pues fijaos, yo conozco sonidos muy divertidos. En mi habitación, por ejemplo, la puerta chirría así «krrrrr krac»". Todos los niños rieron al oírla imitar el sonido y el desánimo inicial se fue rompiendo. Ahora, la imitación de sonidos se convirtió en foco de interés, en vez del "introducirlos en la maleta" y ocupó a los niños. La resistencia inicial fue superada y la situación salvada. Por lo tanto, ¿qué fue lo que capacitó a la estudiante para actuar de manera resuelta en vez de darse por vencida o quedarse desconcertada y buscando ayuda? ¿Lo había aprendido durante sus estudios? ¿Ya había adquirido experiencia en el trato con niños? ¿Disponía de talento empático? Estas son preguntas abiertas.

Al dirigirme a la estudiante, me di cuenta de que ella no era consciente de que había salvado e influido positivamente por su actuar en la situación. Sus acciones le parecieron naturales. Los otros tres estudiantes eran, de todas formas, conscientes de su propia desorientación y le estuvieron muy agradecidos a su compañera de estudio por su intervención espontánea.

Esta situación me dio qué pensar porque tenía la impresión de que las estudiantes estaban mal preparadas para esa visita de los niños en la universidad. Me generó un cambio de perspectivas en el sentido de ¿cómo se sienten los niños cuando les invitamos aquí y qué hay que tener en cuenta acerca del recibimiento y el procedimiento?, ¿qué es lo más importante en el trato con niños, en especial, con aquellos tan jóvenes? Todo eso parecía mal preparado. La consciente sensación empática demuestra el conocimiento sobre el significado del depender el uno del otro, aquí relacionado con la convivencia pedagógica. Incluso la más detallada preparación técnica sirve de nada, si no está dirigida a los niños/adolescentes quienes deben disfrutar de la oferta. El ajuste fino generalmente solo se puede hacer en la situación misma, en la línea del "knowing in action" (Van Manen, 1995b), mediante la buena observación de los niños: ¿Cómo están sentados en el círculo? ¿Parecen optimistas ante la actividad planeada o están, más bien, tímidos? ¿Tengo que hacer algo para aumentar su sensación de seguridad antes de continuar con lo previsto?, etc. Comprender a los niños requiere empatía, así como la capacidad de identificar el estado de ánimo de un grupo, pero también de reconocer las necesidades individuales y tenerlas en cuenta para el actuar pedagógico profesional. La verdadera comprensión de su modo de pensar y de su imaginación, lo que expresan, por ejemplo, mediante su lenguaje y sus cuentos, requiere una capacidad de observación bien entrenada, la capacidad de escuchar activamente y experiencia en el trato con niños. La comprensión pedagógica, o 
sea comprender a los niños, es una capacidad que no se puede dar, por supuesto, en el caso de los jóvenes interesados en los estudios de ciencias de la educación. Es un conocimiento pedagógico que se debe tematizar en relación con la empatía y que se debe transmitir a fondo.

El ejemplo arriba citado muestra que, en el trato pedagógico con niños, es esencial la comprensión de la percepción de los niños y que eso requiere la capacidad empática, la cual lee los sentimientos, comprende el lenguaje y sabe cambiar de perspectiva. Eso hay que destacarlo en la educación docente.

Los siguientes aspectos pueden ayudar a integrar la empatía en la educación docente como conocimiento pedagógico:

a) Educación en valores y, sobre esta base, la reflexión sobre la intención de la educación

b) Experiencia(s) práctica(s) en el trato con niños (aún no en la enseñanza misma) y su reflexión

c) Aprendizaje por medio de estudios de caso

d) Practicar la escucha activa y la comunicación sin violencia

e) Contemplación y entrenamiento de la percepción

A continuación se detalla cada uno de estos aspectos:

a) Educación en valores y, sobre esta base, la reflexión sobre la intención de la educación

Los profesores se enfrentan a presiones sociales de expectativas bastante elevadas: se espera de ellos que introduzcan a los niños y jóvenes en la cultura social de hoy y en las técnicas relevantes, así como que los preparen para los retos del mañana. Deben familiarizarles con los contenidos educativos relevantes y asegurar que se aplique un fundamento democrático de valores comunes que preserva, asegura y promueve la cohesión social. En este ámbito genuinamente pedagógico de tareas y exigencias, el papel de las ciencias educativas debe ofrecer orientación como mediar entre las diferentes exigencias y necesidades arriba mencionadas. Para el actuar pedagógico y los procesos educativos puestos en marcha, es esencial preguntarse cuál es el fundamento de valores sobre el que se realiza o puede realizarse el primero y cuáles son los objetivos/intenciones (MüllerUsing, 2013). A propósito de este tema, la empatía puede proporcionar indicaciones muy útiles. Sin la base de conocimientos de esta, los valores y sus interpretaciones están por sí solos; únicamente en relación con una 
comprensión básica de la empatía (sentido común en cuanto a las relaciones humanas) se incorporan en un proceso dialogal que facilita un crecimiento mutuo y, a través de esto, aprendizaje y formación.

b) Experiencia(s) práctica(s) en el trato con niños (aún no en la enseñanza misma) y su reflexión

Una posibilidad es, por ejemplo, ofrecer un semestre pedagógico de prácticas, acompañado por la universidad, que permita aprender sobre el trato con niños y reflejarlo en un contexto académico. Sería el primer semestre, al comienzo de cada carrera pedagógica. La vivencia demuestra que muchos estudiantes de pedagogía, similar al ejemplo de arriba, apenas han ganado experiencia en el trato con niños y ni siquiera consiguen cumplir con los requisitos más básicos de cada día. Para crear una base sólida y asegurar cierto grado de comprensión básica del comportamiento y actuar infantil, podría concebirse un semestre dirigido a ganar formación docente como de la pedagogía elemental. La reflexión de la experiencia práctica debería ser asegurada por la universidad.

\section{c) Aprendizaje por medio de estudios de casos}

La casuística pedagógica y el aprendizaje de casos pedagógicos son elementos cruciales de la profesionalización de futuros profesores. Al volver la mirada a las grandes novelas educativas (Erziehungsromane) de la Ilustración (Rousseau, Salzmann, más tarde Pestalozzi, etc.), estas representan una forma original del conocimiento pedagógico. Como tales, constituyen una parte integral de la formación docente académica. Trabajar en casos implica ocuparse de la historia de educación de un individuo o un grupo. En la pedagogía escolar, las situaciones suelen venir del contexto de esta y señalan una circunstancia o un problema que puede producirse en la vida escolar profesional. En los estudios de casos, se puede tanto presentar como analizar avances educativos y problemas escolares típicos de ciertos niños, el comportamiento de los profesores, escenas de una reunión de padres de alumnos o bien discusiones entre profesores. Según la experiencia de diez años en el archivo de casos de la Universidad de Kassel, ese trabajo contribuye a hacer más tangible la educación docente y permite la formación de experiencias realistas del trabajo de los profesores mientras la presión de actuar desaparece. Facilita un manejo competente de la teoría, el empirismo y la práctica; ofrece un fórum de discusión sobre interpretaciones y enfoques de acción y puede ayudar a encontrar modos alternativos de proceder. 


\section{d) Practicar la escucha activa y la comunicación sin violencia}

Un requisito central para la transformación de empatía en comportamiento prosocial es la capacidad de escuchar con mucha atención; así se revela el sentido de las palabras. En un área determinada por la comunicación, este aspecto debería considerarse de gran importancia también durante la formación, sobre todo porque una comunicación consciente y el acto de aprender, de escucharse y comprenderse ya incluye gran parte de una empatía vivida (Rogers, 2005; Rosenberg, 2003).

\section{e) Contemplación y entrenamiento de la percepción}

El último aspecto que quiero mencionar es la contemplación y el entrenamiento de la percepción. Educadores y profesores apenas tienen la posibilidad de retirarse y reflexionar durante su día laboral. Son presentes cada día con su ser integral y la necesidad de reaccionar y aprender de cumplir adecuadamente, ante las múltiples exigencias que se ven obligados a enfrentar. Una profesora finlandesa resumió muy bien estas exigencias diarias en una entrevista Müller-Using (2010) cuando declara:

Yo creo que este es uno de los trabajos o labores más desafiantes; el trabajo más desafiante que yo pueda imaginar, ya que no hay límites precisos para lo que se puede hacer. Quizá solo nuestra salud y nuestra propia energía ponen los límites. Como qué podemos hacer... cuánta energía podemos encontrar en nosotros mismos y dársela a los niños: esto es lo único de las principales cosas que determinan lo que podemos hacer. Y este es un trabajo muy demandante, porque la única herramienta para llevarlo a cabo es nuestra propia personalidad. Es muy demandante si, por ejemplo, tenemos un mal día y no debemos transparentarlo a los alumnos, ya que no es su culpa si nos estamos sintiendo tristes algún día. Bueno, si estamos felices supongo que eso se verá; los alumnos pueden fácilmente leernos, pues ya han aprendido a conocernos muy bien y ellos saben qué clase de día estamos teniendo. Por lo tanto, es muy difícil, pues nuestra personalidad es la principal herramienta que tenemos. (p. 178)

[I think this is one of the most challenging jobs or work, the most challeging work I can imagine, because there are no limits what you can really do. Probably your own health and your own energy only make the limits. Like what you can... how much energy you can find from yourself and to give to the children and that's the only one of the main things which controls over what you can do. And this is a very demanding job because the only tool to do this is actually your own personality. So it's really sort of demanding if you happened to have a bad day I shouldn't still show it to my pupils, because 
it's not their fault if I'm feeling sad someday. Well if I'm happy I suppose it will show, the pupils can easily read me they learned to know me very well and they know what kind of a day I do really have. So it's really hard because your personality is the main tool that you really have.] (Entrevista original en inglés)

Este enunciado subraya el significado de la propia personalidad para la profesión docente. A largo plazo, sin embargo, la preocupación por el bienestar de los demás, en este caso de los alumnos, debe ir acompañado de la preocupación por uno mismo. La conciencia y el conocimiento de sí mismo, del acceso individual al aprendizaje (ligado a la capacidad sensorial individual), pueden ayudar a reconocer y fomentar la sensibilidad hacia los niños y sus necesidades, así como los accesos sensoriales al aprendizaje que priorizan. La empatía y el comportamiento empático lo apoyan, en el sentido de que cuanto más sabemos de nuestras propias necesidades y nuestros puntos fuertes o débiles, tanto mejor podemos percibir, reconocer y ayudar a desarrollar los de los niños y adolescentes.

\section{4. ¿La empatía como parte del conocimiento no discursivo?}

Conocimientos no discursivos son aquellas formas del saber que, por ejemplo, según Langer (1992), pasan por conocimiento presencial codificado (e. g. las artes como la música) o, de acuerdo con Polanyi (1985), por conocimiento implícito (e. g. intuición y "sudden insights"). Paschen (2005;2009) y Van Manen (19911995) traducen esta escuela de pensamiento para el sector educativo e introducen formas de conocimiento recién reconocidas, como el conocimiento experiencial e incorporado (Storch, Cantieni, Hüther y Tschacher, 2010; Wilkening, 2011) en el discurso educativo (filosófico).

En mi opinión, hay numerosos indicios de que la empatía forma parte de la categoría del conocimiento no discursivo o implícito. Rifkin (2012) y De Waal (2009) la describen como el hilo conductor de la evolución humana o bien como el fundamento del comportamiento humano elemental, opinión que comparte la investigación en neonatos y la psicología del desarrollo (Bischof-Köhler, 2011). Con base en el sentido común que fue desarrollándose en el curso de la evolución y en el conocimiento de que dependemos unos de otros, la empatía parece ser una de las condiciones previas para la comprensión mutua entre los individuos. Por eso, la capacidad empática del personal docente es un concepto educativo central, porque concierne la misma interacción pedagógica que significa ajustar y equilibrar los procesos de la (auto) educación y la instrucción. 
Van Manen describe el término insight como una categoría de conocimiento separada, una del conocimiento no discursivo o bien implícito. Es un conocimiento disponible y, si se utiliza de manera profesional, permite que la persona actuante tome decisiones, en segundos, sobre lo pedagógicamente favorable o no, lo bueno o malo, lo correcto o falso, lo adecuado o inadecuado. Se puede tratar de un conocimiento experiencial, individual o colectivo e incluso de uno incorporado. El uso activo de estas áreas de conocimiento permite al docente un ajuste de su actuar, inclusive durante el proceso en curso; con esto se comprueba y asegura que el actuar pedagógico sea adecuado (Muñoz, 2009), por ejemplo, con respecto a la intención y la reacción:

Actuar con tacto como educador puede significar, en una situación particular, ser capaz de ver lo que ocurre con los niños, entender la experiencia de los niños, captar la significación pedagógica de esta situación, saber qué hacer y cómo y, realmente, hacer algo en forma correcta.

[To act tactfully as an educator may mean, in a particular situation, to be able to see what goes on with children, to understand the child's experience, to sense the pedagogical significance of this situation, to know how and what to do, and actually to do something right.] (Texto original en inglés)

Según las descripciones de Van Manen, el tacto pedagógico va acompañado de un alto nivel de capacidad empática, que, en mi opinión, constituye la base del tacto pedagógico.

Las explicaciones arriba expuestas muestran que los procesos de coordinación pedagógica y la realización de métodos adaptivos e individuales de enseñanza deberían hacerse sobre la base de la empatía, que implica razonamientos tanto abstractos como autoreflexivos y, por eso, va más allá del simple proceso de sentir con los demás. Mediante la empatía, las intenciones de los demás no solo se pueden sentir, también percibir y comprender cognitivamente, lo que permite reaccionar de manera adecuada al comportamiento del otro que se dirige en cierta dirección. La relevancia pedagógica de este fenómeno es obvia. Si hablamos ahora de la empatía como componente del saber pedagógico, podemos dar por supuesto que se trata de capacidades empáticas fundamentales que permiten encontrar la buena manera (tacto) de comportarse con alumnos individuales, con grupos de ellos o, como $\mathrm{Mu}$ ñoz (2009) lo pondría, para darles a estos una educación adecuada.

Puesto que el saber profesional docente y, conjuntamente, el pedagógico siempre forman parte del conocimiento sobre el actuar profesional, incluyen tanto el 
saber teórico como el conocimiento práctico sobre el actuar pedagógico (Baumert y Kunter, 2006; Bromme, 2004; Neuweg, 2005). Por un lado, el saber pedagógico profesional está ligado al conocimiento experiencial, por ejemplo, a casos, episodios y manuscritos. A la hora de ponerlo en práctica, sin embargo, debe estar tan flexible que permita realizar con éxito el ajuste fino intuitivo en el momento mismo del actuar. Por tanto, el trabajo con casos pedagógicos es de importancia particular para la educación docente.

Como lo han expuesto Baumert y Kunter (2006), entre otros, en el marco de su investigación internacional, hay muy pocos datos válidos de estudio en esta área temática, la que sin duda es muy relevante para la pedagogía, pero "suave" desde ese punto de vista investigativo. Así, siguen existiendo grandes inseguridades con respecto al trato pedagógico apropiado con alumnos, por ejemplo, acerca del modo de instruirlos y de orquestar oportunidades de aprendizaje. Cambiar esto es una de las metas de este trabajo y de mi investigación. Según mi experiencia, sin embargo, para conseguir un verdadero cambio, los futuros profesores deben ser preparados aún mejor para manejar y reflejar estas situaciones pedagógicas que requieren un alto grado de capacidad empática y de ajuste a las necesidades del alumno.

"La educación del niño debe estar dirigida a desarrollar plenamente la personalidad, las habilidades, y las capacidades físicas y mentales del niño". Esto no solo es comprender una educación humanista del siglo de la Ilustración, sino, a la vez, derecho internacional vigente, pues así fue como se aprobó el 20 de noviembre de 1989 en la Declaración de los Derechos del Niño de las Naciones Unidas, reconocida y ratificada en ciento noventa y cinco países miembros, entre ellos Alemania y Costa Rica (Naciones Unidas, 1989). Pero este derecho a la educación no desarrolla su impacto concreto en los niños desde el papel en el cual se escribió, sino solo cuando maestros profesionales y comprometidos, respetados y con un corazón abierto, lo cumplen diariamente en sus clases y aulas. Este tiene que ser el punto de partida y la medida de cualquier educación con perspectiva humanista, ajustada a las condiciones del siglo XXI.

\section{Referencias}

Aust, S., Alkan Hartwig, E., Heuser, I y Bajbouj, M. (2013). The Role of Early Emotional Neglect in Alexithymia. Psychological Trauma, 5(3), 225-232.

Batson, D., Ahmad, N., Powell, A y Stocks, L. (2008). Prosocial motivation. En Shah, J. y Gardner, W. (2007). Handbook of motivational Science pp. 135-149), New York: The Guilford Press. 
Batson, D., Duncan, B., Ackerman, P., Buckley, T y Birch, K. (1981). Is empathic emotion a source of altruistic motivation? Journal of Personality and Social Psychology, 40 (2), 290-302.

Bauer, J. (2005). Warum ich fühle, was du fühlst intuitive Kommunikation und das Geheimnis der Spiegelneuron. Hamburg: Hoffman und Campe.

Bauer, J. (2006). Prinzip Menschlichkeit. Warum wir von Natur aus kooperieren. Hamburg: Hoffmann und Campe.

Baumert, J. y Kunter, M. (2006). Stichwort. Professionelle Kompetenz von Lehrkräften. ZfE, 47, 469-520.

Bertelsmann Stiftung. (2007). Lehrer unter Druck. Arbeitsplatz Schule: zwischen Sokrates und Sozialarbeit. Gütersloh: Bertelsmann Stiftung.

Bischof-Köhler, D. (2011). Soziale Entwicklung in Kindheit und Jugend. Bindung, Empathie, Theory of Mind. Stuttgart: Kohlhammer.

Breithaupt, F. (2009). Kulturen der Empathie. Frankfurt: Suhrkamp.

Breithaupt, F. (2017). Die dunklen Seiten der Empathie. Frankfurt: Suhrkamp.

Breyer, T. (2013). Grenzen der Empathie. Philosophische, psychologische und anthropologische Perspektiven. München: Klostermann.

Brockhaus Wissensservice. (2014). Empathie. Recuperado de https://ub-osnabrueck. brockhaus.de/

Bromme, R. (2004). Das implizite Wissen des Experten. En B. Koch-Priewe, F., Kolbe-Using y J. Wildt. (2004). Grundlagenforschung und mikrodidaktische Reformansätze zur Lehrerbildung, Bad Heilbrunn: Klinkhardt.

De Waal, F. (2009). Das Prinzip Empathie. Was wir von der Natur für eine bessere Gesellschaft lernen können, München: Carl Hanser.

Fehsbach, D. (1978). Studies of empathic behavior in children. En Maher, A. (1964). Progress in experimental personality research, 1-47, New York: Academic Press Inc.

Fend, H. (2006). Neue Theorie der Schule. Wiesbaden: VS Verlag.

Fröhlich, D. (2002). Wörterbuch Psychologie. München: dtv.

Hart, S. y Hodson, V. (2010). Empathie im Klassenzimmer. Ein Lehrern und Lernen, das zwischenmenschliche Beziehungen in den Mittelpunkt stellt, Paderborn: Junfermann. 
Hoffend, A. (2010). Pädagogisches Verstehen. Eine Herausforderung für die Lehrerbildung. Münster: Waxmann.

Keysers, C. (2012). The empathic brain. How the discovery of mirror neurons changes our understanding of human nature. Lexington: Social Brain Press.

Kuhl, J., Müller-Using, S. y Solzbacher, C. (2011). Bildung braucht Beziehung: Selbstkompetenz stärken - Begabungen entfalten. Freiburg: Herder.

Langer, S. (1992). Philosophie auf neuen Wegen. Das Symbol im Denken, im Ritus und in der Kunst. Frankfurt: Suhrkamp.

Lebort, S. (2008). Verlorene Emotionen. Wissenschaftsmagazin fundiert. Recuperado de http://www.fuberlin.de/presse/publikationen/fundiert/archiv/2008_01/08_01_lebort/index.html

Levinas, E. (1995). Zwischen uns. Versuche über das Denken an den Anderen. München: Carl Hanser.

Mayer, A. (2013). Grenzen der Empathie im Angesicht von Opazität. En Breyer, T. (2013). Grenzen der Empathie. Philosophische, psychologische und anthropologische Perspektiven. München: Klostermann, 109-134, Aemania: Wilhelm Fink Verlag.

Melzer, W. y Al-Diban, S. (2001). Vermittlung von Fachleistungs-, Sozial- und Selbstkompetenzen als zentrale Bildungsaufgabe von Schule. En Melzer, W. y Sandfuchs,U. (2001). Was Schule leistet: Funktionen und Aufgaben von Schule, 37-64. Weinheim: Juventa.

Müller-Using, S. (2010). Ethos und Schulqualität. Pädagogisch-ethische Aspekte im professionellen Umgang mit SchülerInnen in Dänemark, Finnland und Deutschland. Opladen: Budrich UniPress.

Muñoz, V. (2009). Vortrag zum Recht auf Bildung des UN-Sonderberichterstatters für das Recht auf Bildung, Prof. Vernor Muñoz, am 7. Juni 2009 in Oldenburg (Oldb) in der Vortrags- und Podiumsveranstaltung, Bildung ist ein Recht und keine Ware - Für eine Bildung gleich hoher Qualität für alle“ im städtischen Kulturzentrum PFL. Recuperado de http://www.munoz.uri-text.de

Muth, J. (1962). Pädagogischer Takt. Monographie einer aktuellen Form erzieherischen und didaktischen Handelns. Heidelberg: Quelle und Meyer.

Myschker, N. y Stein, R. (2014). Verhaltensstörungen bei Kindern und Jugendlichen: Erscheinungsformen - Ursachen - hilfreiche Maßnahmen, Stuttgart: Kohlhammer.

Nano Spezial. (2014). Amok. Recuperado de http://www.3sat.de/page/?source=/nano/gesellschaft/175379/index.html (21.07.2014). 
Neuweg, Georg H. (2005). Emergenzbedingungen pädagogischer Könnerschaft. En Harteis, C, (Hrsg). Verwertbarkeit. Ein Qualitätskriterium (erziehungs-) wissenschaftlichen Wissens? Wiesbaden, 205-228.

Paschen, H. (2005). Zur Entwicklung menschlichen Wissens. Die Aufgabe der Integration heterogener Wissensbestände. Münster: Lit Verlag.

Pfetsch, J., Müller, C. y Itte, A. (2014). Cyberbullying und Empathie. Affektive, kognitive und medienbasierte Empathie im Kontext von Cyberbullying im Kindes- und Jugendalter. In: Diskurs Kindheits- und Jugendforschung, 9 (1), 23-37.

Polanyi, M. (1985). Implizites Wissen.Frankfurt: Suhrkamp Verlag KG

Popp, U. (2012). Individuelle Förderung und die Gestaltung von Zeit- und Lernräumen. En Solzbacher, C., Müller-Using, S., Doll, I. (Eds.). Ressourcen stärken. Individuelle Förderung als Herausforderung für die Grundschule. München, 389-401.

Prange, K. (2005). Die Zeigestruktur der Erziehung. Grundriss der Operativen Pädagogik. Paderborn: Schöningh.

Rifkin, J. (2012). Die empathische Zivilisation. Wege zu einem globalen Bewusstsein. Frankfurt: Fischer.

Rizzolatti, G y Sinigaglia, C. (2008). Empathie und Spiegelneurone: die biologische Basis des Mitgefühls, Frankfurt: Suhrkamp.

Rogers, C. (2005). Die klientenzentrierte Gesprächspsychotherapie. Frankfurt: Fischer.

Rosenberg, M. (2003). Gewaltfreie Kommunikation. Paderborn: Junfermann.

Sekretariat der Ständigen Konferenz der Kultusminister der Länder in der Bundesrepublik Deutschland (KMK). (2004). Standards für die Lehrerbildung: Bildungswissenschaften. Beschluss der Kultusministerkonferenz vom 16.12.2004. Recuperado de http://www.kmk.org/fileadmin/veroeffentlichungen_beschluesse/2004/2004_12_16-Bildungsstandards-Konzeption-Entwicklung.pdf (21.7.2014).

Sekretariat der Ständigen Konferenz der Kultusminister der Länder in der Bundesrepublik Deutschland (KMK). (2014). Schulgesetze der Länder in der Bundesrepublik Deutschland. Recuperado de http://www.kmk.org/dokumentation/rechtsvorschriften-und-lehrplaene-der-laender/uebersicht-schulgesetze.html (21.7.2014).

Schweer, M. y Padberg, J. (2002). Vertrauen im Schulalltag: eine pädagogische Herausforderung. Neuwied: Luchterhand.

Shi, Z. (2011). Multisensorische Wahrnehmung. Recuperado de http://www.psy.lmu.de/ exp/people/ma/shi/docs/s1_ohne_bg.pdf (15.08.2014). 
Singer, T. y Bolz, M. (2013). Mitgefühl in Alltag und Forschung. München: Max Planck Society.

Solzbacher, C., Behrensen, B., Sauerhering M., Schwer, C. (2012). Jedem Kind gerecht werden? Sichtweisen und Erfahrungen von Grundschullehrkräften, Köln: Carl Link Verlag.

Storch, M., Cantieni, B., Hüther, G. y Tschacher, W. (2010). Embodiment. Die Wechselwirkung von Körper und Psyche verstehen und nutzen. Bern: Hans Huber.

Schulz von Thun, F. (2011). Miteinander reden (1-3). Sonderausgabe, Reinbek: Rowohlt.

Universität Kassel. (2014). Fallarchiv Schulpädagogik.. Recuperado de http://www.fallarchiv.uni-kassel.de

Van Manen, M. (1991). The tact of teaching. The meaning of pedagogical thoughtfulness. SUNY series, the philosophy of education, Albany: State University of New York Press.

Van Manen, M. (1995a). Herbart und der Takt im Unterricht. In: Anonymous Didaktik und/ oder Curriculum. Zeitschrift für Pädagogik. Weinheim.Beltz, 61-80

Van Manen, M. (1995b). On the Epistemology of Reflective Practice. Teachers and teaching 1 (1), University of Alberta, 33-50.

Von Hentig, H. (2009). Das Ethos der Erziehung. Was ist in ihr elementar? En Zeitschrift für Pädagogik. Weinheim Beltz, 55 (4), 509-527.

Wilkening, F. y Cacchione, T. (2011). Children's intuitive physics. En Goswami, U. (Ed.): The Wiley- Blackwell Handbook of childhood cognitive development, 2nd ed. Chichester. 473-496.

Wolfgang, N. (2002). Kompetenz en Otto, H., Rauschenbach, T. Y y Vogel, P. (2002). Erziehungswissenschaft: Professionalität und Kompetenz, Opladen,13-27.

Zumbach, J., Haider, K. y Mandl, H. (2008). Fallbasiertes Lernen: Theoretischer Hintergrund und praktische Anwendung. En Zumbach, J. y Mandl, H. Pädagogische Psychologie in Theorie und Praxis: Ein fallbasiertes Lehrbuch. Göttingen: Hogrefe, 1-11. 